\title{
Desenvolvimento vegetativo de híbrido de orquídea em diferentes substratos
}

\section{Vegetative development of orchid hybrid in different substrates}

\author{
Alessandro Garrett Dronk ${ }^{1}$; Alexandre Peterson Vieira da Silva ${ }^{2}$; \\ Francine Lorena Cuquel'3; Ricardo Tadeu de Faria ${ }^{4 *}$
}

\begin{abstract}
Resumo
A produção de orquídeas é uma atividade em crescente expansão nos mercados nacional e internacional, tornando-se evidente a necessidade de informações que permitam a otimização do seu cultivo. Para a produção comercial de orquídeas, é imprescindível a utilização de um substrato que permita maior crescimento das mudas e, na seleção deste material, é muito importante avaliar aspectos do ponto de vista econômico, físico, químico, biológico e ecológico. O objetivo deste trabalho foi avaliar o desenvolvimento de clones de BLC Nan Chang Silk 'Olimpic Torch', obtida do cruzamento entre BLC. Bryce Canyon x BLC. Pamela Hetherington, em diferentes substratos. Os substratos avaliados foram: T1: fibra de xaxim (controle); T2: fibra de coco; T3: casca de pinus compostado; T4: carvão vegetal; T5: casca de pinus compostado + fibra de coco $(1: 1 \mathrm{v} / \mathrm{v})$; T6: fibra de coco + carvão vegetal $(1: 1 \mathrm{v} / \mathrm{v})$; T7: carvão vegetal + casca de pinus compostado $(1: 1 \mathrm{v} / \mathrm{v})$; T8: carvão vegetal + casca de pinus compostado + fibra de coco $(1: 1: 1 \mathrm{v} / \mathrm{v} / \mathrm{v})$. As mudas foram cultivadas em vasos de polipropileno, permanecendo em viveiro com $80 \%$ de luminosidade. Os parâmetros avaliados 24 meses após o início do experimento foram: número de pseudobulbos, diâmetro médios dos pseudobulbos, altura da parte área da planta, relação altura da parte aérea /comprimento dos pseudobulbos e o $\mathrm{pH}$ e a condutividade elétrica dos substratos. Com base nos parâmetros de desenvolvimento vegetativos concluiu-se que todos os substratos avaliados podem ser utilizados eficientemente para o cultivo de BLC Nan Chang Silk 'Olimpic Torch'.
\end{abstract}

Palavras-chave: Propagação, Orchidaceae, plantas ornamentais

\begin{abstract}
For commercial production of orchids, it is essential to use a good substrate, and the selection of this material is very important to evaluate aspects of the economic, physical, chemical, biological, and ecological points of view. The aim of this study was to evaluate the development of clones of BLC Nan Chang Silk 'Olimpic Torch' on different substrates. The substrates were: T1: tree fern fiber "xaxim" (control), T2: coconut fiber, T3: composted pine bark, T4: plant coal, T5: composted pine bark + coconut fiber $(1: 1 \mathrm{v} / \mathrm{v})$ T6: coconut fiber + plant coal $(1: 1 \mathrm{v} / \mathrm{v})$, T7: plant coal + composted pine bark $(1: 1 \mathrm{v} / \mathrm{v})$, T8: plant coal + composted pine bark + coconut fiber $(1: 1: 1 \mathrm{v} / \mathrm{v} / \mathrm{v})$. The seedlings were grown in pots of polypropylene, kept in a nursery with $80 \%$ of brightness. We evaluated 24 months after the start of the experiment: number of pseudobulbs, the lenght of pseudobulbs, height of the plant area, height of the aerial part / lenght of the pseudobulbs and $\mathrm{pH}$ and electrical conductivity of the substrate. Based on the parameters of vegetative development it was concluded that all the evaluated substrates can be efficiently used for the cultivation of BLC Nan Chang Silk 'Olimpic Torch'.
\end{abstract}

Key words: Propagation, Orchidaceae, ornamental plants

\footnotetext{
${ }^{1}$ Eng $^{\mathrm{o}}$ Agr ${ }^{\mathrm{o}}$, M. Sc em Agronomia, Universidade Federal do Paraná, UFPR, Curitiba, PR. E-mail: orquidronk@yahoo.com.br

${ }^{2}$ Eng $^{\circ}$ Agr $^{\circ}$, M. Sc em Agronomia, UEL, Londrina, PR. E-mail alexandrepeterson@gmail.com

${ }^{3}$ Prof. Dr. em Fitotecnia, UFPR, Curitiba, PR, PR. E-mail: francine@ufpr.br

${ }^{4}$ Prof. Dr. em Genética, Universidade Estadual de Londrina, UEL, Londrina, PR. E-mail: faria@uel.br

* Autor para correspondência
} 


\section{Introdução}

A produção de orquídeas é uma atividade em crescente expansão nos mercados nacional e internacional, tornando-se evidente a necessidade de informações que permitam a otimização do seu cultivo (LORENZI; SOUZA, 2001).

Espécies de Cattleya e outros gêneros da família Orchidaceae são muito cultivados no mundo inteiro como plantas ornamentais pela beleza de suas flores, o que lhes confere grande importância econômica. O gênero Cattleya engloba cerca de 70 espécies e inúmeras variedades e híbridos. São encontradas como nativas desde as regiões do México, América Central até a América do Sul (SORACE et al., 2009). Constitui um dos mais belos ornamentos das matas tropicais e subtropicais da América, tornando se o mais popular e o mais cultivado gênero da família das orquídeas (RAPOSO, 1993).

Para a produção comercial de orquídeas, é imprescindível a utilização de um substrato que permita maior crescimento das mudas e, na seleção deste material, é muito importante avaliar aspectos do ponto de vista econômico, físico, químico, biológico e ecológico. Sob o aspecto econômico, o substrato deve ser encontrado facilmente e possuir baixo valor comercial (YAMAKAMI et al., 2006; VILLA et al., 2007). Nos aspectos físicos e químicos as qualidades básicas e indispensáveis de um substrato são: consistência para suporte, boa aeração das raízes, capacidade de retenção de água, $\mathrm{pH}$ adequado, entre outras (SILVA; SILVA, 1997; KÄMPF, 2000; SILVA, 2000; SOUZA, 2003). As orquídeas epífitas cultivadas em recipientes desenvolvem-se melhor em substratos de textura relativamente grossa e de drenagem livre, proporcionando às raízes livre acesso ao ar e à luz, como ocorre na natureza (BICALHO, 1969).

O xaxim, foi o substrato preferido pela maioria dos orquidófilos brasileiros até (1992) quando sua utilização foi proibida pois as plantas fornecedoras deste material (Dicksonia sellowiana), encontramse em processo de extinção, devido ao extrativismo desenfreado, apesar da legislação do meio ambiente em vigor (IBAMA, 1992). Esse substrato é considerado excelente para o cultivo de orquídeas, pois retém grande volume de água, conservando-se úmido por longo tempo (SILVA, 1986).

Pesquisando substratos alternativos para Oncidium sarcodes e Schomburgkia crispa, (REGO et al., 2000) e, para Oncidium baueri e Maxillaria consangüinea, Faria et al. (2001) concluíram que o xaxim pode ser substituído por outros substratos. $\mathrm{O}$ uso de substratos alternativos para o cultivo de orquídeas traz uma série de benefícios à natureza, entre eles a preservação da $D$. sellowiana (LORENZI; SOUSA, 2001). Segundo Silva (1986) e Colombo et al. (2005), outros materiais como: raízes de Polypodium (espécie de samambaia), fibra e raízes de Osmunda regalis (samambaiareal), casca de barbatimão, casca de pinus, fibra de coco, carvão vegetal e argila expandida podem ser utilizados no cultivo de orquídeas epífitas.

O carvão vegetal é um substrato alternativo, pois apresenta uma estrutura bastante porosa e, misturado a outros materiais como fibra de coco, casca de arroz carbonizada e casca de pinus, aumenta a porosidade, a capacidade de retenção de água, facilitando a proliferação de microrganismos benéficos (SCHNITZER et al., 2010).

Apesar de existir uma grande diversidade de materiais que podem ser utilizados como substrato alternativo e misturas no cultivo de orquídeas o seu sucesso depende da espécie e do tipo de ambiente onde será efetuado o cultivo (COOKE, 1999).

O objetivo deste trabalho foi avaliar o desenvolvimento de clones de BLC Nan Chang Silk 'Olimpic Torch', obtida do cruzamento entre $B L C$. Bryce Canyon x BLC. Pamela Hetherington, em diferentes substratos.

\section{Material e Métodos}

O experimento foi conduzido durante 24 meses, no Orquidário Garrett Dronk em Curitiba (PR), 
localizada nas coordenadas $25^{\circ} 26^{\prime} 07.52$ 'S e $49^{\circ}$ 18 ' 48.85" e altitude de $898 \mathrm{~m}$ em relação ao nível do mar, cuja classificação climática segundo Köppen (1948) é Cfb.

Os clones de orquídea BLC Nan Chang Silk 'Olimpic Torch', obtida do cruzamento entre BLC. Bryce Canyon x BLC. Pamela Hetherington, no inicio do experimento, com dois anos de idade, apresentavam três pseudobulbos cada, com diâmetro de 4,2 $\pm 0,5 \mathrm{~cm}$, altura da parte aérea 6,5 $\pm 0,3 \mathrm{~cm}$ e a relação entre a altura da parte aérea e o comprimento do pesudobulbo de $1,55 \mathrm{~cm}$.

As plantas foram cultivadas em vasos de polipropileno com volume de $166,9 \mathrm{~cm}^{3}$, em estufa com $80 \%$ de retenção de luminosidade, obtida através de uma tela de polipropileno preta. A irrigação foi efetuada no período matutino, sendo diária no verão e a cada três dias no inverno, até a capacidade do vaso. As adubações foram feitas com o adubo foliar N:P:K (10-10-10) na dose de 1 $\mathrm{g} \mathrm{L}^{-1}$, sendo aplicados $50 \mathrm{~mL}$ desta solução por vaso a cada trinta dias. A cada três meses, foi efetuada uma adubação orgânica, utilizando farinha de osso e torta de mamona e cinzas $(1: 1: 1 \mathrm{v} / \mathrm{v} / \mathrm{v})$, sendo adicionado $1 \mathrm{~g} \mathrm{vaso}^{-1}$ (SILVA, 1986).

Os substratos estudados foram: T1: fibra de xaxim; T2: fibra de coco; T3: casca de pinus compostado; T4: carvão vegetal; T5: casca de pinus compostado + fibra de coco $(1: 1 \mathrm{v} / \mathrm{v})$; T6: fibra de coco + carvão vegetal $(1: 1 \mathrm{v} / \mathrm{v})$; T7: carvão vegetal + casca de pinus compostado $(1: 1 \mathrm{v} / \mathrm{v})$; T8: carvão vegetal + casca de pinus compostado + fibra de coco $(1: 1: 1 \mathrm{v} / \mathrm{v} / \mathrm{v})$.

Ao final do experimento foram analisadas as seguintes características: número de pseudobulbos (NP), comprimento dos pseudobulbos (DP), altura da parte área $(\mathrm{H})$ e a relação altura da parte aérea pelo comprimento do pseudobulbo (H/CP).
Em relação ao substrato foram determinados o $\mathrm{pH}$ e a condutividade elétrica. $\mathrm{O} \mathrm{pH}$ em água foi medido por meio de eletrodo combinado imerso em suspensão substrato: água ( $1: 2,5 \mathrm{v} / \mathrm{v})$, metodologia adaptada da Embrapa (1997), e a condutividade elétrica dos substratos foi medida por meio de um condutivímetro em suspensão de substrato : água $(1: 1 \mathrm{v} / \mathrm{v})$ (CAMARGO et al., 1986).

O delineamento experimental utilizado foi inteiramente casualizado, com oito tratamentos e doze repetições por tratamento. Cada repetição foi constituída por uma planta. Os dados foram submetidos a analise de variância, seguida pelo Teste de Tukey a 5\% de probabilidade, com auxilio dos software Microsoft Excel e SASM-Agri (CANTERI et al., 2001).

\section{Resultados e Discussão}

Conforme apresentado na Tabela 1, não houve efeito significativo dos substratos para nenhuma das características avaliadas, mostrando a eficiência dos substratos estudados em substituição a utilização do xaxim. O uso de substratos alternativos ao xaxim para orquídeas epífitas também foi relatado por Faria et al (2001) usando vermiculita para Oncidium baueri e para Maxillaria picta usando vermiculita + carvão e vermiculita + casca de arroz carbonizada em proporções iguais; Rego et al. (2000) para orquídea Oncidium sarcodes obtiveram os melhores resultados nos substratos: casca de pinus + isopor + carvão; vermiculita + casca de arroz carbonizada + carvão + isopor; casca de pinus e casca de pinus + carvão, sendo as misturas realizadas em proporções iguais e para Schomburgkia crispa os substratos: casca de pinus + isopor + carvão; vermiculita + casca de arroz carbonizada + carvão + isopor e casca de pinus. 
Tabela 1. Médias do número de pseudobulbos (NP), comprimento de pseudobulbo (CP), altura da parte aérea $(\mathrm{H})$ e relação entre altura da parte aérea e comprimento de pseudobulbos (H/CP) de clones de BLC Nan Chang Silk 'Olimpic Torch' obtida do cruzamento entre BLC. Bryce Canyon x BLC. Pamela Hetherington cultivadas em diferentes substratos, após vinte e quatro meses do início do experimento. Curitiba, 2009.

\begin{tabular}{|c|c|c|c|c|}
\hline \multirow[b]{2}{*}{ Tratamentos } & \multicolumn{3}{|c|}{ Pseudobulbos } & \multirow[b]{2}{*}{$\mathrm{H} / \mathrm{CP}(\mathrm{cm})$} \\
\hline & NP & $\mathbf{C P}(\mathrm{cm})$ & $\mathbf{H}(\mathbf{c m})$ & \\
\hline T1: fibra de xaxim (controle) & 5,50 a & $13,11 \mathrm{a}$ & 21,80 a & $1,71 \mathrm{a}$ \\
\hline T2: fibra de coco & 5,08 a & 13,28 a & 20,37 a & 1,57 a \\
\hline T3: casca de pinus compostado & 5,42 a & $12,68 \mathrm{a}$ & 21,43 a & 1,72 a \\
\hline T4: carvão vegetal & 5,08 a & 13,21 a & 22,88 a & 1,78 a \\
\hline T5: casca de pinus compostado + fibra de coco & 5,42 a & 12,43 a & $21,47 \quad a$ & 1,75 a \\
\hline T6: fibra de coco + carvão vegetal & 5,08 a & $12,73 \mathrm{a}$ & 23,45 a & 1,89 a \\
\hline T7: carvão vegetal + casca de pinus compostado & 5,42 a & $12,97 \mathrm{a}$ & 22,17 a & 1,75 a \\
\hline $\begin{array}{l}\text { T8: carvão vegetal + casca pinus compostado + } \\
\text { fibra de coco }\end{array}$ & 5,83 a & 12,85 a & 23,43 & 1,86 a \\
\hline C.V.(\%) & 14,65 & 8,7 & 15,99 & 22,18 \\
\hline
\end{tabular}

Médias seguidas da mesma letra nas colunas não diferem entre si pelo teste de Tukey, a 5\% de significância.

Fonte: Elaboração dos autores.

Sorace et al. (2009) observaram que xaxim desfibrado, pó de bagaço de cana-de-açúcar, pó de bagaço de cana-de-açúcar: isopor picado $(1: 1, \mathrm{v}: \mathrm{v})$; pó de bagaço de cana-de-açúcar: casca de pinus (1:1, v:v); pó de bagaço de cana-de-açúcar: pó de coco (1:1, v:v) podem ser utilizados como substitutos ao xaxim no cultivo do híbrido Miltonia regnellii Rchb. f. X Oncidium concolor Hook. Colombo et al. (2005) usaram como substrato pó de coco para o híbrido da orquídea Cattleya chocolate drop $\mathrm{x}$ (C. guttata $x$ L. tenebrosa). Segundo Schinitzer et al. (2010), o carvão vegetal também pode ser utilizado como substrato ou entrar na composição de misturas trazendo benefícios não somente para desenvolvimento vegetativo da planta como de micorrizas.

Para as características altura da planta, número e comprimento de pseudobulbo não houve diferença significativa entre os substratos. As plantas apresentaram um desenvolvimento compatível com a idade das plantas (4 anos) e a relação altura e comprimento de pseudobulbo variou entre 1,57 e $1,89 \mathrm{~cm}$, não diferindo estaticamente (Tabela 1).

Os dados referentes ao $\mathrm{pH}$ e à condutividade elétrica dos substratos encontram-se na Tabela 2. Quanto ao parâmetro $\mathrm{pH}$, os diferentes substratos apresentam resultados estatisticamente distintos, sendo o carvão vegetal o que apresentou maior resultado (valor de 5,95) em variações de $\mathrm{pH}$ como estas, também foram observada por Sorace et al. (2009), em cultivos do $C$. intermedia $X$ Hadrolaelia purpurata, que variaram de 3,70 a 5,65 nos substratos: xaxim desfibrado (testemunha); casca de arroz carbonizada; fibra de coco; casca de pinus; casca de pinus + fibra de coco $(1: 1 \mathrm{v} / \mathrm{v})$; casca de arroz + fibra de coco $(1: 1 \mathrm{v} / \mathrm{v})$; casca de arroz carbonizada + casca de pinus $(1: 1 \mathrm{v} / \mathrm{v})$; casca de pinus + casca de arroz carbonizada + fibra de coco $(1: 1: 1 \mathrm{v} / \mathrm{v} / \mathrm{v})$. 
Tabela 2. Médias do pH e condutividade elétrica $(\mathrm{CE})\left(\mu \mathrm{S} . \mathrm{cm}^{-1}\right)$ dos substratos utilizados no cultivo de clones de $B L C$ Nan Chang Silk 'Olimpic Torch', após dos vinte e quatro meses do inicio do experimento. Curitiba, 2009.

\begin{tabular}{lrrrr}
\hline & \multicolumn{1}{c}{ Tratamentos } & pH & \multicolumn{2}{c}{$\mathbf{C E}(\boldsymbol{\mu S}$..cm } \\
\hline
\end{tabular}

Médias seguidas da mesma letra nas colunas não diferem entre si pelo teste de Tukey, a 5\% de significância.

Fonte: Elaboração dos autores.

Segundo Röber e Schaller (1985) e Kämpf (2000), a faixa ideal de $\mathrm{pH}$, para o cultivo de Cattleya é de 5,0 a 5,5. Entretanto, como foi observado, tanto o valor de $\mathrm{pH}$ de 3,49 , quanto o valor de 5,95 (Tabela 2) apresentaram-se favoráveis para o desenvolvimento das plantas (Tabela 1).

A variação da condutividade elétrica, nos substratos testados, ficou entre $65,58 \mu \mathrm{S} . \mathrm{cm}^{-1}$, em casca de pinus e $213,95 \mu \mathrm{S} . \mathrm{cm}^{-1}$ em carvão, diferindo entre si estatisticamente conforme observado na Tabela 2. Segundo Takane, Faria e Altafin (2006), valor de condutividade superior a $500,00 \mu \mathrm{S} / \mathrm{cm}$, caracteriza salinização do substrato, é elevado para orquídeas epífitas, podendo causar perda de água pelas raízes, ocasionando manchas ou queimas visíveis nas folhas. No entanto, apesar de ser observada diferença estatística entre os substratos no parâmetro condutividade elétrica, os demais parâmetros que avaliavam desenvolvimento vegetativo (Tabela 1), mostram que a não houve diferença estatística. Wang e Gregg (1994) realizaram trabalhos variando a condutividade elétrica em Phalaenopsis sp. e observaram que não houve influência no desenvolvimento das plantas.

Graciano et al. (1995) e Handrech e Black (1999), em seu estudo apresentam duas causas de variação da condutividade elétrica do substrato, sendo elas a adubação de base ou do conteúdo natural de sais presentes no substrato. Como a adubação do experimento foi igual para todos os substratos, as diferenças na condutividade elétrica observada (Tabela 2), possivelmente foram causadas pelo conteúdo natural de sais dos substratos e a capacidade de retenção de cátions dos substratos.

\section{Conclusão}

Conclui-se que todos os substratos analisados podem ser utilizados como substratos alternativos ao xaxim para o cultivo de BLC Nan Chang Silk 'Olimpic Torch'.

\section{Referências}

BICALHO, H. D. Subsídios à orquidocultura paulista. Bol. Inst. Bot., São Paulo, n. 6, 1969.

CAMARGO, O. A.; MONIZ, A. C.; JORGE, J. A.; VALADARES, J. M. A.S. Métodos de análise química, mineralógic e física de solos do Instituto Agronômico de Campinas. Campinas: Instituto Agronômico, 1986. (Boletim técnico, 106).

CANTERI, M. G.; ALTHAUS, R. A.; VIRGENS FILHO, J. S.; GIGLIOTI, E. A.; GODOY, C. V. SASM - Agri: sistema para análise e separação de médias em experimentos agrícolas pelos métodos Scoft - Knott, Tukey e Duncan. Revista Brasileira de Agrocomputação, Londrina, v. 1, n. 2, p. 18-24, 2001. 
COLOMBO, L. A.; FARIA, R. T.; ASSIS, A. M.; FONSECA, I. C. B. Aclimatização de um híbrido de Cattleya em substratos de origem vegetal sob dois sistemas de irrigação. Acta Scientiarum, Maringá, v. 27, n. 1, p. 145-150, jan./mar. 2005.

COOKE, R. B. Estufas e telados. Revista Oficial do Orquidário, Rio de Janeiro, v. 13, n. 3-4, p. 94-101, 1999.

EMPRESA BRASILEIRA DE PESQUISA AGROPECUÁRIA - EMBRAPA. Manual de métodos de análise de solos. Centro Nacional de Pesquisa de Solos. Rio de Janeiro: Embrapa, 1997.

FARIA, R. T.; REGO, L. V.; BERNARDI, A.; MOLINARI, H. Performance of diferents genotyps of brazilian orchid cultivation in alternatives substrates. Brazilian Archives of Biology and Technology, Curitiba, v. 44, n. 4, p. 337-342, 2001.

GRACIANO, T.; DEMATTE, J.; VOLPE, C.; PERECIN, D. Interação entre substratos e na germinação e na produção de mudas Tagetes patula L.(compositae). Revista Brasileira de Horticultura Ornamental, Campinas, v. 1, n. 2, p. 78-85, 1995.

HANDRECH, K. A.; BLACK, N. Growing media for ornamental plants and flowers. Sydney: University of New South Wales Press, 1999.

IBAMA. Lista oficial das espécies da flora ameaçadas de extinção. Diário Oficial. Portaria 006/92-N. de 15 de jan. 1992.

KÄMPF, A. N. Produção comercial de plantas ornamentais. Guaíba, RS: Agropecuária, 2000.

KÖPPEN, W. Climatologia; com um estudiu de los climas de la tierra. México: Fondo de Cultura Económica, 1948. $479 \mathrm{p}$.

LORENZI, H. S.; SOUZA, H. M. Plantas ornamentais no Brasil. 3. ed. Nova Odessa: Ed. Plantarum Ltda, 2001. $835 \mathrm{p}$.

RAPOSO, J. G. C. M. F. A etimologia a serviço dos orquidófilos. São Paulo: Ave Maria Ltda, 1993.

REGO, L. V.; BERNARDI, A.; TAKAHASHI, L. S. A.; FARIA, R. T. Desenvolvimento vegetativo de genótipos de orquídeas brasileiras em substratos alternativos ao xaxim. Revista Brasileira de Horticultura Ornamental, Campinas, v. 6, n. 1-2, p. 75-79, 2000.
RÖBER, R.; SCHALLER, K. Pflanzenernährung im gartenbau. Stuttgart: Ulmer, 1985.

SCHNITZER, J. A.; FARIA, R. T.; VENTURA, M. U.; SORACE, M. Substratos e extrato pirolenhoso no cultivo de orquídeas brasileiras Cattleya intermedia (John Lindley) e Miltonia clowesii (John Lindley) (Orchidaceae). Acta Scientiarum Agronomy, Maringá, v. 32, n. 1, p. 139-143, 2010.

SILVA, F. S. C. Haverá algum substrato que substitua o xaxim? Boletim da Coordenadoria das Associações Orquidófilas do Brasil (CAOB), Rio de Janeiro, n. 44, p. 68-76, 2000.

SILVA, F. S. C.; SILVA, S. P. C. O substrato na cultura das orquídeas, sua importância, seu envelhecimento. Revista Oficial do Orquidário, Rio de Janeiro, v. 11, n. 1, p. 3-10, 1997.

SILVA, W. Cultivo de orquídeas no Brasil. São Paulo: Nobel, 1986.

SORACE, M.; FARIA, R. T.; FONSECA, I. C.; YAMAMOTO, L. Y.; SORACE, M. A. F. Substratos alternativos ao xaxim no cultivo do híbrido Cattleya intermedia X Hadrolaelia purpurata (Orchidaceae), Semina: Ciências Agrárias, Londrina, v. 30, n. 4, p. 771778, 2009.

SOUZA, M. Muito além do xaxim. Natureza, São Paulo, v. 1, n. 2, p. 32-37, 2003.

TAKANE, R. J.; FARIA, R. T.; ALTAFIN, V. L. Tecnologia fácil - 75: cultivo de orquídeas. Brasília: LK, 2006.

VILLA, F.; PEREIRA, A. R.; PASQUAL, M.; ARAUJO, A. G. Influência de substratos alternativos na aclimatização de orquídea. Revista Ceres, Viçosa, v. 54, n. 316, p. 501-505, 2007.

WANG, Y. T.; GREGG, L. L. Médium and fertilizer affect the performance of Phalaenopsis during two flowers cycles. HortScience, Alexandria, v. 29, n. 4, p. 269-270, 1994.

YAMAKAMI, J. Y.; FARIA, R. T.; ASSIS, A. M.; REGOOLIVEIRA, L. V. Cultivo de Cattleya (Orchidaceae) em substratos alternativos ao xaxim. Acta Scientiarium Agronomy, Maringá, v. 28, n. 4, p. 523-526, 2006. 